\section{Catecholaminergic polymorphic} ventricular tachycardia in 2012

Ineke Nederend, Christian van der Werf, Arthur A.M. Wilde

Department of Cardiology, Heart Failure Research Center, Academic Medical

Center, Amsterdam, The Netherlands

\section{Abstract}

Catecholaminergic polymorphic ventricular tachycardia (CPVT) is a rare, potentially lethal inherited arrhythmia syndrome characterized by stress or emotion-induced ventricular arrhythmias. CPVT was first described in 1960 , while the genetic basis underlying this syndrome was discovered in 2001. The past decade has seen substantial advances in understanding the pathophysiology of CPVT. In addition, significant advances have been made in elucidating clinical characteristics of CPVT patients and new treatment options have become available. Here, we review current literature on CPVT to present state-of-the-art knowledge on the subject of the genetic basis, pathophysiology, clinical presentation, diagnosis, treatment and prognosis.

\section{Introduction}

Catecholaminergic polymorphic ventricular tachycardia (CPVT) is a malignant inherited arrhythmia syndrome. The main pathologic feature is adrenergic-induced bidirectional or polymorphic ventricular tachycardia (VT) in the absence of organic heart disease and apparent 12-lead ECG abnormalities. ${ }^{1}$ CPVT is a relatively new entity.

The first report on patients with the clinical characteristics of CPVT was described by Berg in $1960 .^{2}$ CPVT was definitely acknowledged as a primary arrhythmia syndrome in 1995 after a patient series described by Leenhardt et al. ${ }^{1}$ The genetic basis underlying CPVT was discovered ten years ago $0^{3-5}$ In recent years, significant progress has been made in understanding the genetic basis, pathophysiology and phenotype of CPVT and how to apply this knowledge in the clinical management of patients and families with CPVT. This review aims to provide an update on current state-of-the-art knowledge regarding CPVT.

\section{Genetic basis}

Whereas a genetic component in CPVT was recognized since its first description, Swan et al. set the first step in identifying the genetic basis of CPVT. ${ }^{6}$ They described two unrelated families including multiple individuals with the typical CPVT phenotype. Genetic linkage analysis assigned the disease locus on a region on the long (q) arm of chromosome 1. However, no mutations or polymorphisms were indentified by sequencing putative candidate genes.

In 2001 this group ${ }^{4}$ and Priori et al. ${ }^{3}$ demonstrated that mutations in the gene encoding the human cardiac ryanodine receptor (RyR2) underlie CPVT. Priori et al. identified RyR2 missense mutations in four out of 12 probands with a strong CPVT phenotype (S2246L, R2474S, N4104K, and R4497C). ${ }^{3}$ Three mutations were de novo, whereas the fourth mutation cosegregated with the phenotype in four relatives of the proband. Laitinen et al. identified a RyR2 missense mutation in three large families that fully cosegregated with the phenotype as well (P2328S, Q4201R, and V4653F). ${ }^{4}$

RyR2 is a 105-exon-containing gene residing on chromosome 1 (1q42-q43). The majority of mutations in RyR2 are missense mutations. Mutations tend to be clustered in three hotspots: the N-terminal domain (aminoacids 77-466), the central domain (aminoacids 2246-2534), and the channel region (aminoacids 3778-4959) (Figure 1). ${ }^{7}$ This phenomenon is the reason why using a tiered strategy in RyR2 sequencing, starting with those exons known to host most unique mutations, is currently the most reasonable approach. ${ }^{7}$ This will probably change in the near future, when high-throughput sequencing instruments will become available and provide whole-gene sequencing in a single step.

Whereas almost all RyR2 mutations seem to result in a classic CPVT phenotype, some exceptions exist. In 2007 our group reported a more complex phenotype caused by a large genomic deletion that involved RyR2 exon-3. ${ }^{8}$ Sixteen carriers from two separate families showed decreased left ventricular function leading to dilated cardiomyopathy, progressive atrioventricular block, sinoatrial node dysfunction, atrial fibrillation, and atrial standstill. In addition, several families with RyR2 missense mutations and discrete right ventricular abnormalities have been identified..$^{9,10}$

A rare, recessively inherited CPVT phenotype was first described by Lahat et al. in $2001 .^{11}$ This report included 9 children who died suddenly and 13 surviving children with a CPVT phenotype, from seven related Bedouin families. In a subsequent report in 2001, this group reported mutations in cardiac calsequestrin (CASQ2) to underlie this autosomal recessive form of CPVT (D307H). ${ }^{5}$

To date, the prevalence mutations in RyR2
Correspondence: Arthur A.M. Wilde, Academic Medical Center, University of Amsterdam, Department of Cardiology, Heart Failure Research Center, Meibergdreef 9, 1105 AZ Amsterdam, The Netherlands.

Tel. +31.0.205.663.072 - Fax. +31.0.206.962.609.

E-mail: a.a.wilde@amc.uva.nl

Key words: catecholaminergic polymorphic ventricular tachycardia; sudden cardiac death; genetics; channelopathies.

Acknowledgments: this work was supported by ZorgOnderzoek Nederland Medische Wetenschappen (ZonMW, grant 120610013 to C.W. and A.A.M.W.), and by the Fondation Leducq TransAtlantic Network of Excellence, Preventing Sudden Death (grant 05-CVD-01 to A.A.M.W.).

Conflicts of interests: the authors declare no potential conflicts of interests.

Authors' contributions: the authors contributed equally.

Received for publication: 28 September 2011. Accepted for publication: 14 November 2011.

This work is licensed under a Creative Commons Attribution NonCommercial 3.0 License (CC BYNC 3.0).

(C) Copyright I. Nederend et al., 2011

Licensee PAGEPress, Italy

Cardiogenetics 2011; 1(s1):e4

doi:10.4081/cardiogenetics.2011.s1.e4

and $\mathrm{CASQ} 2$ are identified in approximately $60 \%$ and $5 \%$ of CPVT patients, respectively (see Genetic testing).

In 2007, a third, highly malignant autosomal recessive CPVT phenotype was mapped to a 25 Mb interval on chromosome 7p14-p22 in a study including four children from a highly inbred family. ${ }^{12}$ All children showed the classic CPVT phenotype, and eventually three children died suddenly while playing. To date, the causal gene has not been identified.

Finally, Andersen-Tawil syndrome (LQTS type 7), which is caused by mutations in the gene encoding KCNJ2, may resemble the CPVT phenotype (see Differential diagnosis).

\section{Pathophysiology}

Ventricular arrhythmias in CPVT are caused by alterations in a critical element of cardiac excitation-contraction coupling: calciuminduced calcium release (CICR). ${ }^{13,14}$ CICR is initiated by calcium influx through the L-type calcium channels in the sarcolemma (Figure 2). This triggers calcium release from the sarcoplasmic reticulum (SR), the main intracellu- 
lar calcium storage, through opening of RyR2. The released calcium binds to troponin $\mathrm{C}$ and eventually results in muscle contraction. This process ends when SR calcium release is terminated, leading to relaxation. The released calcium is mainly recycled back to the SR by the SR calcium-ATPase (SERCA) pump, or extruded from the cell by the sodium/calcium exchanger (NCX).

CPVT-related variants in RyR2 or CASQ2 cause spontaneous RyR2 openings, leading to spontaneous calcium release from the SR. ${ }^{15,16}$ The subsequent increase in cytosolic calcium activates the electrogenic NCX, which causes a transient inward current. This current eventually produces delayed afterdepolatizations (DAD), which may trigger an action potential and lead to triggered arrhythmias. This process is enhanced especially under conditions of $\beta$-adrenergic stimulation. ${ }^{17,18}$ It has been proposed that this mechanism underlying CPVT principally originates in the HisPurkinje system. ${ }^{19,20}$

Mutations in RyR2 alter the channel regulation mechanism, particularly its sensitivity to calcium activation. A recent study provided data supporting the hypothesis that mutations in RyR2 destabilize the channel by mediation of defective interdomain interaction. ${ }^{21}$ The coupled conformational changes lead to erroneous activation of the channel and spontaneous calcium release.

CASQ2 mutations probably cause CPVT by several independent mechanisms.22 First, CASQ2 is the main calcium buffering protein of the SR, and mutated CASQ2 can result in a loss of Ca buffering. Second, loss of CASQ2 may lead to a reduced direct inhibitory effect on RyR2. Lastly, CASQ2 mutations may lead to remodeling of SR structure and proteins, particularly a reduction of the CASQ2-binding proteins triadin and junctin.

\section{Genotype-phenotype correlation}

To date, only data on genotype-phenotype comparing RyR2 mutation-related and nonRyR2 mutation related CPVT patients (in whom CASQ2 and KCNJ2 were not tested) are available. ${ }^{23,24}$ Priori et al. observed RyR2 mutation-carrying index patients were more often male and became symptomatic at younger age. ${ }^{23}$ Sy et al. observed a higher proportion of symptomatic patients among RyR2 mutationcarrying index patients, but the number of patients was low. ${ }^{24}$ CPVT patients carrying mutations in CASQ2 are thought to have a worse phenotype, with expression at younger age, compared to CPVT patients with RyR2 or no mutations. ${ }^{11,25}$
While different RyR2 mutated transgenic and knock-in mouse models show similar phenotypes, ${ }^{26,27}$ genotype-phenotype correlations based on RyR2 mutation location have not been studied in humans.

\section{Clinical characteristics}

\section{Clinical presentation}

The classic CPVT patient is a young child presenting with syncope triggered by exercise and/or emotion, usually accompanied by a family history of sudden cardiac death (SCD) at young age. ${ }^{1}$ Eventually, aborted cardiac arrest (ACA) or SCD under similar circumstances may occur. Patients may be misdiagnosed with epilepsy, because syncope can be accompanied by convulsive movements. ${ }^{28,29}$

In recent years it has been observed that probands presenting at older age are not uncommon. Recently, a distinction between juvenileonset CPVT and late-onset CPVT has been proposed by Sy et al. ${ }^{24}$ This group classified these two groups using a cut-off age of 21 years. The yield of genetic testing was significantly higher in the juvenile-onset group, whereas probands in the late-onset group were more likely to be female and were less likely to have affected family members. This could implicate that the pathophysiological substrate between these two subtypes is different. However, in RyR2 mutation-carrying relatives onset of symptoms may occur at older age, and that does not make it two different types of diseases.

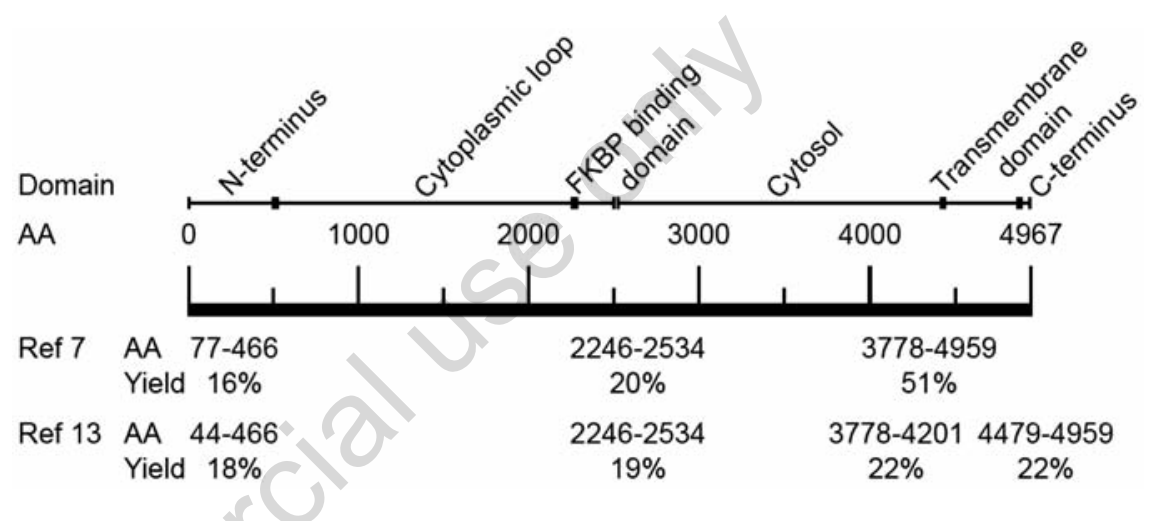

Figure 1. RyR2 structure and mutation clustering. Yield indicates the proportion of RyR2 mutations identified per domain (indicated by the aminoacid number estimated for each domain). AA, amino acid. Adapted from Medeiros-Domingo et al. ${ }^{7}$ and Priori et al. ${ }^{13}$

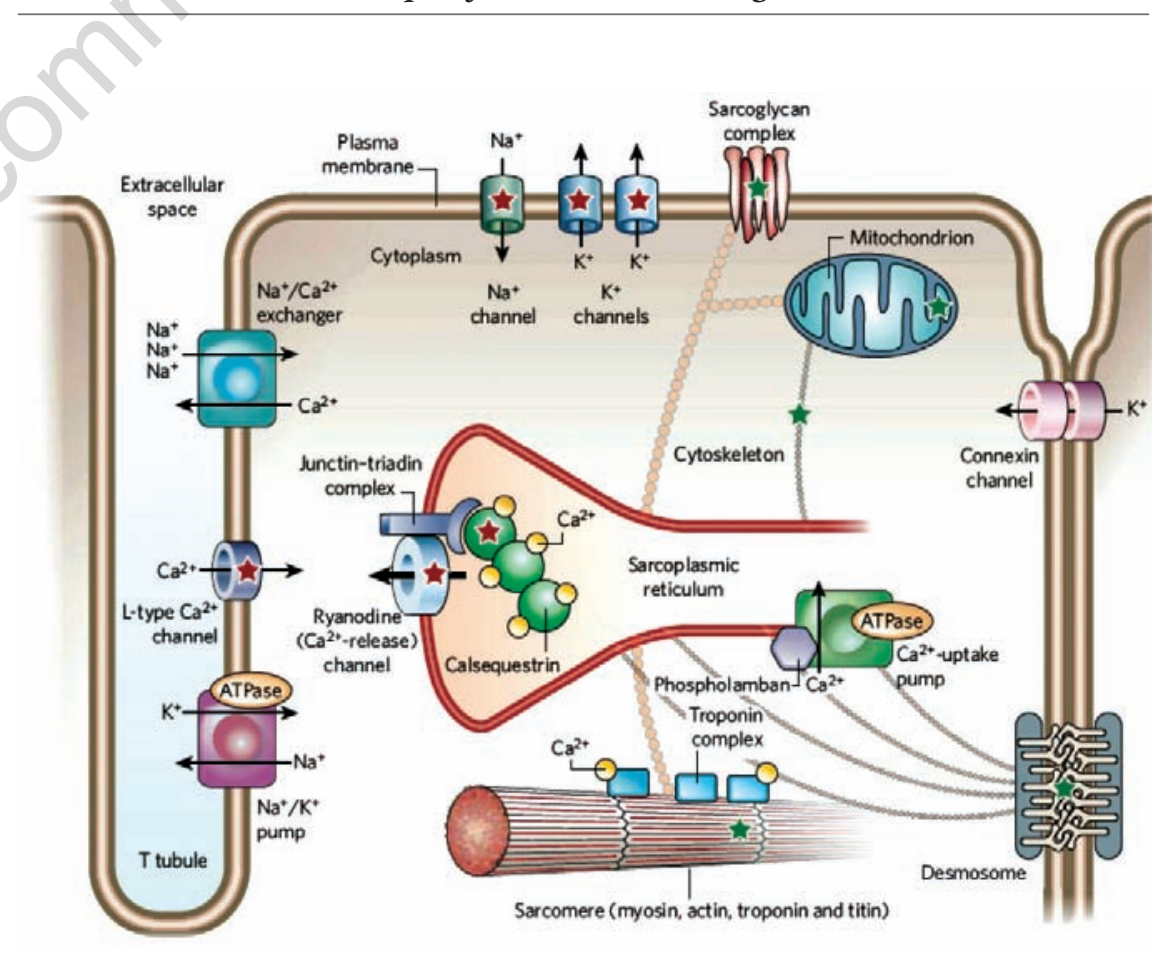

Figure 2. Calcium homeostasis in a ventricular cardiomyocyte. Adapted from Knollmann et al. ${ }^{14}$ with permission. 
Most CPVT cases are related to familial RyR2 mutations, and cascade screening of the CPVTcausing mutation in the proband may lead to the identification of more mutation-carrying relatives. Thus, it is conceivable that among all CPVT patients known to date, mutation-carrying relatives are predominant. However, data on disease penetrance and expression in mutation-carrying relatives are scarce. In one study on 30 RyR2 mutation-carrying relatives, eight (27\%) reported CPVT-related symptoms. ${ }^{30}$ During exercise testing 23 relatives (77\%) showed signs of disease penetrance.

\section{Electrocardiographic characteristics}

CPVT patients show a tendency towards having sinus bradycardia (Figure $3 \mathrm{~A}$ ). ${ }^{31}$ In a patient series including RyR2 mutation-carrying CPVT patients from our group, resting heart rate of 12 probands was 20 beats per minute lower as compared to matched controls. $^{32}$ In addition, symptomatic mutation-carrying relatives had a lower resting heart rate than asymptomatic family members. Sinus bradycardia was more pronounced in males. Two possible explanations were postulated: an impaired calcium ion handling by mutated RYR2 channels in sino-atrial node cells, or vagally-mediated effect through a feedback mechanism that lowers the likelihood of reaching the detrimental threshold at which ventricular arrhythmia starts. The exact underlying mechanism of sinus bradycardia in CPVT has not been clarified. In some patients' resting ECGs prominent U-waves have been observed. ${ }^{33,34}$

Ventricular arrhythmias tend to initiate at an individually fixed and reproducible threshold, usually between 110 and 135 beats per minute. ${ }^{30}$ Typically ventricular arrhythmias evolve from single isolated ventricular premature beats (VPB) to bigeminy, couplets and non-sustained polymorphic or bidirectional VT (Figure 3B-D). However, complexity of ventricular arrhythmias may be limited in some patients (see Genetic testing). Polymorphic VT occurs in approximately $60 \%$ of patients, while the proportion of patients with bidirectional VT is much lower. ${ }^{24,31}$ In more than half of the cases the initiating VPB originates from the right ventricular outflow tract. ${ }^{24,31}$

\section{Diagnosis}

\section{Clinical diagnosis}

The clinical diagnosis of CPVT is made when ventricular arrhythmias, in particular bidirectional or polymorphic VT, are recorded under conditions of increased $\beta$-adrenergic drive, and echocardiography and 12-lead ECG show no apparent abnormalities and thereby reasonably rule out structural heart disease. Exercise testing may be considered as the gold standard diagnostic test in CPVT. In patients with or suspected for long QT syndrome (in particular type 1) exercise testing may also trigger ventricular arrhythmias, but ventricular ectopy beyond isolated VPBs is much more common (87.5\%) in CPVT patients as compared with long QT syndrome patients (7\%). ${ }^{35}$ The presence of bigeminy has a specificity of $96 \%$ and a sensitivity of $81 \%$ for CPVT. ${ }^{35}$

Holter monitoring may be used as an alternative test, for instance in children that are too young to undergo exercise testing, or when a patient's symptoms are more emotion- then exercise-related. Finally, catecholamine infusion may be used to unmask CPVT. ${ }^{24,31}$ Sumitomo et al. observed ventricular arrhythmias induced by exercise testing in all 27 patients, while 12/16 (75\%) had ventricular arrhythmia during catecholamine infusion, and 16/23 (69\%) during Holter monitoring. ${ }^{31}$ However, Sy et al. reported that catecholamine infusion provoked ventricular arrhythmia in three patients who did not show any ventricular arrhythmias on either Holter recordings or exercise testing. ${ }^{24}$ Thus, exercise testing is the first choice diagnostic test, but the diagnostic approach may be tailored to individual patients.

\section{Genetic testing}

In the recent HRS/EHRA Expert Consensus Statement on the State of Genetic Testing for the Channelopathies and Cardiomyopathies, genetic testing in CPVT is recommended for any patient in whom a cardiologist has established a clinical index of suspicion for CPVT based on examination of the patient's clinical history, family history, and expressed electrocardiographic phenotype during provocative stress testing with cycle, treadmill, or catecholamine infusion..$^{36}$ Genetic testing should start with sequencing a limited number of exons in RyR2 (see Genetic basis). In case of a negative result this can be followed by analyzing the remaining RyR2 exons in a stepwise fashion, ${ }^{7}$ and CASQ2. RyR2 mutations are detected in approximately $60 \%$ of cases. However, the exact yield is related to the patient's phenotype (see below). The prevalence of CASQ2 mutations is estimated to be approximately $5 \%$.

Importantly, genetic testing should not be limited to patients meeting the full spectrum of typical marked clinical diagnostic criteria (i.e., the presence of exercise-induced bidirectional or polymorphic VT), because it has been well established that in some patients CPVT may express with less severe ventricular arrhythmias. However, the presence of exercise-induced bidirectional or polymorphic VT is associated with increased odds of identifying a pathogenic mutation: in patients with documented exercise- or emotion-induced bidirectional or polymorphic VT in the absence of structural cardiac disease, RyR2 mutations are detected in $50-70 \% .{ }^{7,37}$ This drops to $38 \%$ in patients with a history of exertion-induced syncope who show ventricular ectopy, but not bidirectional or polymorphic VT, during exercise testing. ${ }^{7}$ One study including a small number of probands suggested that the odds of identifying a RyR2 mutation is higher in younger probands. ${ }^{24}$

The yield of RyR2 genetic testing is $5-31 \%$ in

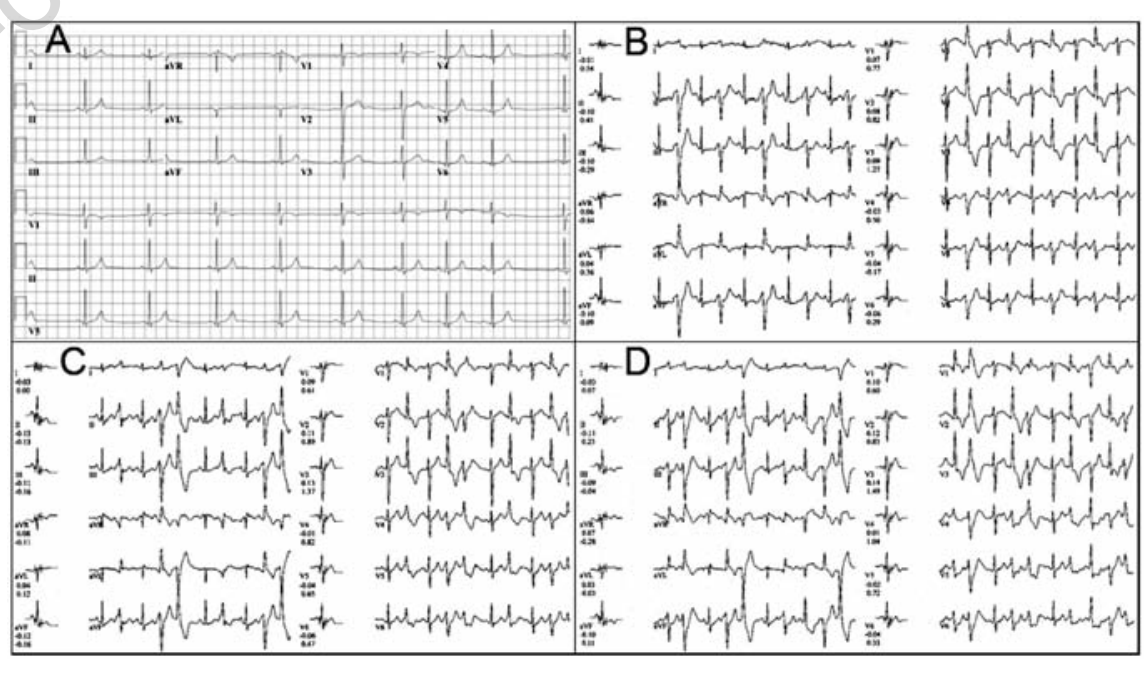

Figure 3. Examples of resting and exercise electrocardiogram (ECG) in a catecholaminergic polymorphic ventricular tachycardia (CPVT) patient. A) Resting ECG showing sinus bradycardia. The U-wave in lead V2 and V3 is of moderate amplitude. B-D) Exercise testing showing increasing complexity of ventricular arrhythmias. Panel B shows ventricular premature beats in bigeminy, panel $\mathrm{C}$ shows polymorphic couplets, and panel $\mathrm{D}$ polymorphic non-sustained ventricular tachycardia of four beats. 
patients with exercise- or emotion- induced syncope without documented ventricular arrhythmias during exercise testing., ${ }^{73}$ Moreover, RyR2 mutations are identified in $15 \%$ of individuals with a normal ECG who are relatives of young SCD or ACA victims when these events were exercise or emotional stress-related. ${ }^{37}$ However, in the absence of (possible) electrocardiographic signs of CPVT genetic testing is not recommended. ${ }^{36}$

RyR2 mutations are also believed to play a role in sudden infant death syndrome (SIDS; unexplained death of an infant under one year of age after extensive postmorten examination). In one study including 134 unrelated SIDS victims two novel RyR2 mutations were detected in two cases. ${ }^{38}$

Finally, the HRS/EHRA Expert Consensus Statement recommends genetic testing for family members and appropriate relatives following the identification of the CPVT-causative mutation in an index case (so-called cascade screening) ${ }^{36}$ As CPVT can manifest at a very young age and RyR2 mutations have been associated with SIDS, cascade screening is recommended at birth. ${ }^{36}$

\section{Differential diagnosis}

In patient presenting with exercise or emotional stress-induced syncope or ACA, LQTS, in particular type 1 , is an alternative diagnosis. When the resting 12-lead ECG is inconclusive, exercise testing can help to discriminate between LQTS and CPVT, as previously discussed (see Clinical diagnosis).

Another differential diagnosis of exerciseinduced bidirectional VT includes AndersenTawil syndrome (LQTS type 7). This condition is usually associated with extracardiac manifestations, i.e. periodic paralysis and facial dysmorphisms, and is caused by loss of function mutations in the gene encoding KCNJ2 gene. $^{39}$

Other alternatives may include (initially concealed) structural heart disease, in particular mitral valve prolapse, arrhythmogenic right ventricular dysplasia/cardiomyopathy, hypertrophic cardiomyopathy and myocardial ischemia.

\section{Clinical management}

Considering the adrenergically mediated nature of the arrhythmias in CPVT, $\beta$-blockers at the maximum tolerable dose are considered the mainstay of treatment. Nadolol is probably most effective. ${ }^{40}$ However, $\beta$-blockers do not completely suppress ventricular arrhythmias in most patients. In addition, it has been well established that a subset of patients are not well protected against arrhythmic events by $\beta$ blockers. In the largest cohort described to date (including 101 patients, of whom 50 were probands) an 8-year arrhythmic event rate of $27 \%$ was observed in patients on $\beta$-blockers. ${ }^{40}$
Recently, we systematically reviewed all studies on efficacy of $\beta$-blockers. ${ }^{41}$ Among the 403 patients included, $88 \%$ were on $\beta$-blockers. We observed 8-year overall, near-fatal, and fatal event rates of $37.2 \%, 15.3 \%$, and $6.4 \%$, respectively (Figure 4). Importantly, some events occurring on $\beta$-blockers may be explained by non-compliance rather than true $\beta$-blocker therapy failure. In one study, patients on $\beta$ blockers showed an earlier onset of ventricular arrhythmias during exercise testing (on the same workload) as compared to baseline, ${ }^{30}$ which would not be a preferable effect. In 2002 Priori et al. observed similar $\beta$-blocker efficacy in RyR2-mutation related and non-RyR2-mutation related CPVT, but event rates in this study were extremely high ${ }^{23}$ and not in line with subsequent observations. ${ }^{41}$

Two studies reported promising results on the use of the L-type calcium channel blocker verapamil. ${ }^{42,43}$ However, on the long term verapamil does not prevent arrhythmic events in all patients. ${ }^{44}$ Verapamil may be most effective in patients carrying CASQ2 mutations. In one
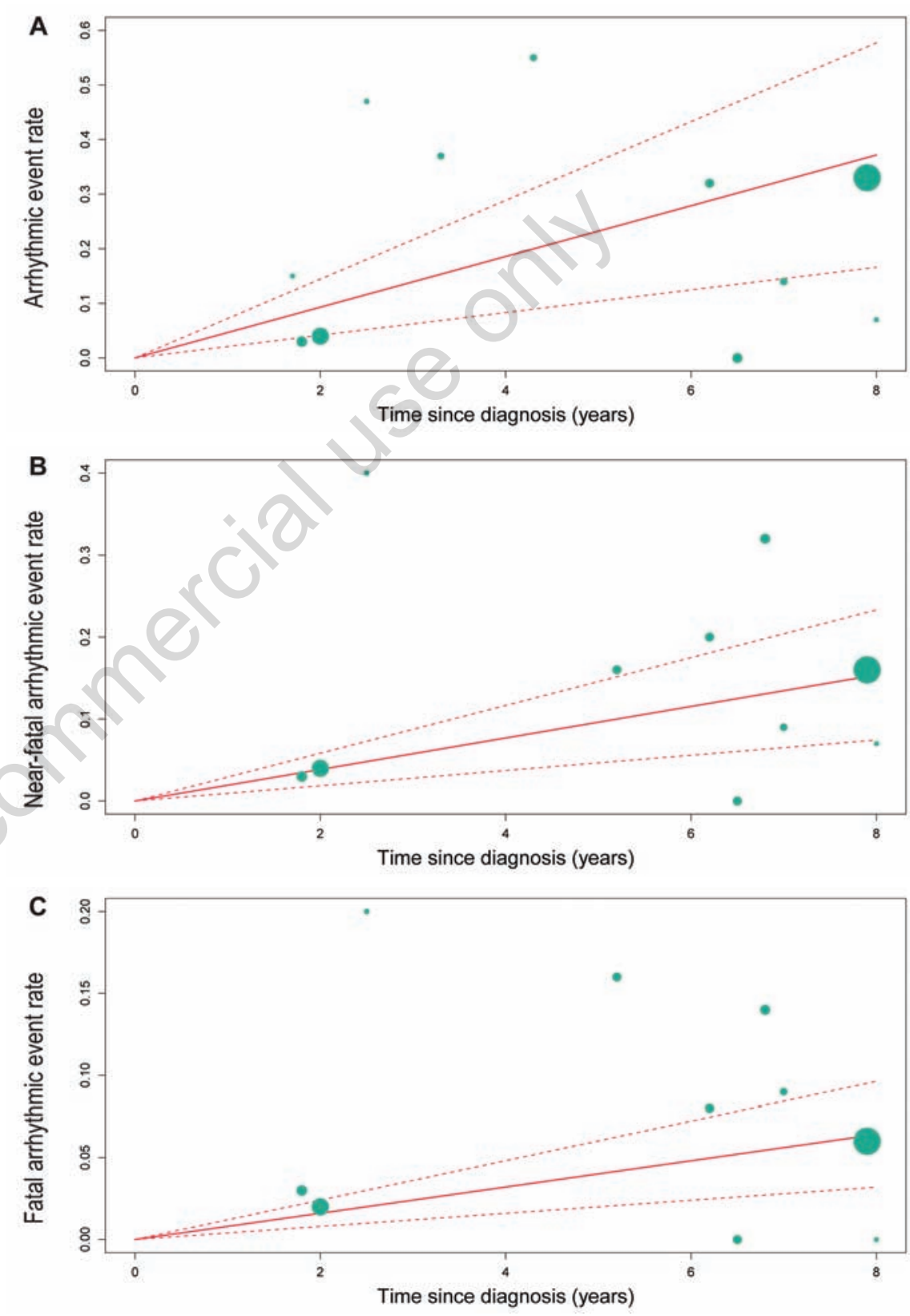

Figure 4. All (A), near-fatal (B) and fatal (C) arrhythmic event curves in catecholaminergic polymorphic ventricular tachycardia (CPVT) patients on $\beta$-blockers therapy. The red line and its corresponding area indicate the proportion of patients with arrhythmic events and its corresponding $95 \%$ confidence interval based on a random-effects meta-analysis model. The green data points represent the arrhythmic event rates of the individual studies. The area of each data point is proportional to its number of patients and statistical weight. Adapted from van der Werf et al., ${ }^{41}$ with permission. 
study, verapamil was the most effective antiarrhythmic drug in reducing ventricular arrhythmia in CASQ2 mutation carrying mice and humans. ${ }^{45}$

Recently, it was discovered that flecainide prevented ventricular arrhythmias in a CASQ2 knock-out mouse model, presumably by directly targeting the underlying defect in CPVT. ${ }^{46}$ Subsequently, we evaluated the efficacy and safety of this flecainide on top of conventional drug therapy in 33 mutation-carrying CPVT patients with continuous symptoms or ventricular arrhythmias. ${ }^{47}$ In 22 patients (76\%) ventricular arrhythmia were partly or completely suppressed $(\mathrm{P}<0.001)$. During a median follow-up of 20 months (range: 12-40) one patient received appropriate ICD shocks, but this event was associated with very low flecainide levels. The other patients remained free of arrhythmic events.

According to current guidelines, ICD implantation is indicated in patients with CPVT who are survivors of cardiac arrest or patients with syncope and/or documented sustained VT despite $\beta$-blocker therapy. ${ }^{48}$ However, in recent year several examples have shown that ICDs may be proarrhythmic in CPVT, as ICDs may trigger ventricular storms by inappropriate or appropriate shocks. ${ }^{28,49,50}$ Indeed, CPVT patients may be at increased risk of inappropriate shocks due to CPVT-related supraventricular arrhythmias. ${ }^{51,52}$

In 2008, the first three CPVT patients with successful prevention of adrenergically-mediated arrhythmias with left cardiac sympathic denervation (LCSD) were described, some with long-term follow-up. ${ }^{53}$ After this, more successful experiences with LCSD in CPVT have been published. ${ }^{54-56}$ Given the excellent results, some authors argue that LSCD should be performed before considering ICD implantation. ${ }^{57}$

Future treatment options may include propafenone, ${ }^{58}$ dantrolene,${ }^{59}$ and further development of the newly developed compounds S107, ${ }^{60}$ K201 (JTV519), ${ }^{61}$ and KN93. ${ }^{62}$

Effectiveness of therapy should be monitored regularly using exercise testing and/or holter monitoring. In addition, discouraging participation in competitive sports and emphasizing therapy compliance may be critical to prevent arrhythmic events. CPVT patients should also be advised not to go swimming alone. ${ }^{3}$

To date, data on risk stratification in CPVT patients are scarce. In particular, predictors for a very low risk for arrhythmias (which would make active treatment unnecessary) have not been identified, as silent mutation carriers are also at risk of arrhythmic events. ${ }^{30,40}$ Thus, based on current knowledge all phenotypically and/or genotypically affected CPVT patients should receive treatment. The only exception may apply for older RyR2-mutation carrying individuals (over approximately 60 years of age) who are identified by cascade screening and have been asymptomatic lifelong.

\section{References}

1. Leenhardt A, Lucet V, Denjoy I, et al. Catecholaminergic polymorphic ventricular tachycardia in children. A 7-year followup of 21 patients. Circulation 1995;91: 1512-9.

2. Berg KJ. Multifocal ventricular extrasystoles with Adams-Stokes syndrome in children. Am Heart J 1960;60:965-70.

3. Priori SG, Napolitano C, Tiso N, et al. Mutations in the cardiac ryanodine receptor gene (hRyR2) underlie catecholaminergic polymorphic ventricular tachycardia. Circulation 2001;103:196-200.

4. Laitinen PJ, Brown KM, Piippo K, et al. Mutations of the cardiac ryanodine receptor (RyR2) gene in familial polymorphic ventricular tachycardia. Circulation 2001; 103:485-90.

5. Lahat H, Pras E, Olender T, et al. A missense mutation in a highly conserved region of CASQ2 is associated with autosomal recessive catecholamine-induced polymorphic ventricular tachycardia in Bedouin families from Israel. Am J Hum Genet 2001;69:1378-84.

6. Swan H, Piippo K, Viitasalo M, et al. Arrhythmic disorder mapped to chromosome 1q42-q43 causes malignant polymorphic ventricular tachycardia in structurally normal hearts. J Am Coll Cardiol 1999;34: 2035-42.

7. Medeiros-Domingo A, Bhuiyan ZA, Tester DJ, et al. The RYR2-encoded ryanodine receptor/calcium release channel in patients diagnosed previously with either catecholaminergic polymorphic ventricular tachycardia or genotype negative, exercise-induced long QT syndrome: a comprehensive open reading frame mutational analysis. J Am Coll Cardiol 2009;54:206574.

8. Bhuiyan ZA, van den Berg MP, van Tintelen JP, et al. Expanding spectrum of human RYR2-related disease: new electrocardiographic, structural, and genetic features. Circulation 2007;116:1569-76.

9. Tiso N, Stephan DA, Nava A, et al. Identification of mutations in the cardiac ryanodine receptor gene in families affected with arrhythmogenic right ventricular cardiomyopathy type 2 (ARVD2). Hum Mol Genet 2001;10:189-94.

10. d'Amati G, Bagattin A, Bauce B, et al. Juvenile sudden death in a family with polymorphic ventricular arrhythmias caused by a novel RyR2 gene mutation: evidence of specific morphological sub- strates. Hum Pathol 2005;36:761-7.

11. Lahat H, Eldar M, Levy-Nissenbaum E, et al. Autosomal recessive catecholamine- or exercise-induced polymorphic ventricular tachycardia: clinical features and assignment of the disease gene to chromosome 1p13-21. Circulation 2001;103:2822-7.

12. Bhuiyan ZA, Hamdan MA, Shamsi ET, et al. A novel early onset lethal form of catecholaminergic polymorphic ventricular tachycardia maps to chromosome 7p14p22. J Cardiovasc Electrophysiol 2007;18: 1060-6.

13. Priori SG, Chen SR. Inherited dysfunction of sarcoplasmic reticulum $\mathrm{Ca} 2+$ handling and arrhythmogenesis. Circ Res 2011;108: 871-83.

14. Knollmann BC, Roden DM. A genetic framework for improving arrhythmia therapy. Nature 2008;451:929-36.

15. Jiang D, Xiao B, Yang D, et al. RyR2 mutations linked to ventricular tachycardia and sudden death reduce the threshold for store-overload-induced $\mathrm{Ca} 2+$ release (SOICR). Proc Natl Acad Sci U S A 2004; 101:13062-7.

16. di Barletta MR, Viatchenko-Karpinski S, Nori A, et al. Clinical phenotype and functional characterization of CASQ2 mutations associated with catecholaminergic polymorphic ventricular tachycardia. Circulation 2006;114:1012-9.

17. Knollmann BC, Chopra N, Hlaing T, et al. Casq2 deletion causes sarcoplasmic reticulum volume increase, premature $\mathrm{Ca} 2+$ release, and catecholaminergic polymorphic ventricular tachycardia. J Clin Invest 2006;116:2510-20.

18. Cerrone M, Noujaim SF, Tolkacheva EG, et al. Arrhythmogenic mechanisms in a mouse model of catecholaminergic polymorphic ventricular tachycardia. Circ Res 2007;101:1039-48.

19. Herron TJ, Milstein ML, Anumonwo J, et al. Purkinje cell calcium dysregulation is the cellular mechanism that underlies catecholaminergic polymorphic ventricular tachycardia. Heart Rhythm 2010;7:1122-8.

20. Kang G, Giovannone SF, Liu N, et al. Purkinje cells from RyR2 mutant mice are highly arrhythmogenic but responsive to targeted therapy. Circ Res 2010;107:512-9.

21. Suetomi T, Yano M, Uchinoumi $\mathrm{H}$, et al. Mutation-linked defective interdomain interactions within ryanodine receptor cause aberrant $\mathrm{Ca} 2+$ release leading to catecholaminergic polymorphic ventricular tachycardia. Circulation 2011;124:68294.

22. Chopra N, Knollmann BC. Cardiac calsequestrin: the new kid on the block in arrhythmias. J Cardiovasc Electrophysiol 2009;20:1179-85.

23. Priori SG, Napolitano C, Memmi M, et al. 
Clinical and molecular characterization of patients with catecholaminergic polymorphic ventricular tachycardia. Circulation 2002;106:69-74.

24. Sy RW, Gollob MH, Klein GJ, et al. Arrhythmia characterization and longterm outcomes in catecholaminergic polymorphic ventricular tachycardia. Heart Rhythm 2011;8:864-71.

25. Postma AV, Denjoy I, Hoorntje TM, et al. Absence of calsequestrin 2 causes severe forms of catecholaminergic polymorphic ventricular tachycardia. Circ Res 2002;91: e21-26.

26. Cerrone M, Colombi B, Santoro M, et al. Bidirectional ventricular tachycardia and fibrillation elicited in a knock-in mouse model carrier of a mutation in the cardiac ryanodine receptor. Circ Res 2005;96:e77-82.

27. Kannankeril PJ, Mitchell BM, Goona-sekera SA, et al. Mice with the R176Q cardiac ryanodine receptor mutation exhibit catecholamine-induced ventricular tachycardia and cardiomyopathy. Proc Natl Acad Sci U S A 2006;103:12179-84.

28. Palanca V, Quesada A, Trigo A, Jimenez J. [Arrhythmic storm induced by AICD discharge in a patient with catecholaminergic polymorphic ventricular tachycardia]. Rev Esp Cardiol 2006;59:1079-80. [Article in Spanish].

29. Rutter N, Southall DP. Cardiac arrhythmias misdiagnosed as epilepsy. Arch Dis Child 1985;60:54-6.

30. Haugaa KH, Leren IS, Berge KE, et al. High prevalence of exercise-induced arrhythmias in catecholaminergic polymorphic ventricular tachycardia mutation-positive family members diagnosed by cascade genetic screening. Europace 2010;12:417-23.

31. Sumitomo N, Harada K, Nagashima M, et al. Catecholaminergic polymorphic ventricular tachycardia: electrocardiographic characteristics and optimal therapeutic strategies to prevent sudden death. Heart 2003;89:66-70.

32. Postma AV, Denjoy I, Kamblock J, et al. Catecholaminergic polymorphic ventricular tachycardia: RYR2 mutations, bradycardia, and follow up of the patients. J Med Genet 2005;42:863-70.

33. Aizawa Y, Komura S, Okada S, et al. Distinct $U$ wave changes in patients with catecholaminergic polymorphic ventricular tachycardia (CPVT). Int Heart J 2006;47:381-9.

34. Viitasalo M, Oikarinen L, Väänänen $H$, et al. U-waves and T-wave peak to T-wave end intervals in patients with catecholaminergic polymorphic ventricular tachycardia, effects of $\beta$-blockers. Heart Rhythm 2008;5:1382-8.

35. Horner JM, Ackerman MJ. Ventricular ectopy during treadmill exercise stress testing in the evaluation of long QT syndrome. Heart Rhythm 2008;5:1690-4.

36. Ackerman MJ, Priori SG, Willems S, et al. HRS/EHRA Expert Consensus Statement on the State of Genetic Testing for the Channelopathies and Cardiomyopathies This document was developed as a partnership between the Heart Rhythm Society (HRS) and the European Heart Rhythm Association (EHRA). Heart Rhythm 2011;8:1308-39.

37. Bai R, Napolitano C, Bloise R, et al. Yield of genetic screening in inherited cardiac channelopathies: how to prioritize access to genetic testing. Circ Arrhythm Electrophysiol 2009;2:6-15.

38. Tester DJ, Dura M, Carturan E, et al. A mechanism for sudden infant death syndrome (SIDS): stress-induced leak via ryanodine receptors. Heart Rhythm 2007; 4:733-9.

39. Barajas-Martinez H, Hu D, Ontiveros G, et al. Biophysical and molecular characterization of a novel de novo KCNJ2 mutation associated with Andersen-Tawil syndrome and catecholaminergic polymorphic ventricular tachycardia mimicry. Circ Cardiovasc Genet 2011:4:51-7.

40. Hayashi M, Denjoy I, Extramiana F, et al. Incidence and risk factors of arrhythmic events in catecholaminergic polymorphic ventricular tachycardia. Circulation 2009; 119:2426-34.

41. van der Werf C, Zwinderman AH, Wilde AA. Therapeutic approach for patients with catecholaminergic polymorphic ventricular tachycardia: state of the art and future developments. Europace 2011. [Epub ahead of print].

42. Rosso R, Kalman JM, Rogowski 0, et al. Calcium channel blockers and $\beta$-blockers versus $\beta$-blockers alone for preventing exercise-induced arrhythmias in catecholaminergic polymorphic ventricular tachycardia. Heart Rhythm 2007;4:114954.

43. Swan H, Laitinen P, Kontula K, Toivonen L. Calcium channel antagonism reduces exercise-induced ventricular arrhythmias in catecholaminergic polymorphic ventricular tachycardia patients with RyR2 mutations. J Cardiovasc Electrophysiol 2005;16: 162-6.

44. Rosso R, Kalman J, Rogowsky 0, et al. Long-term effectiveness of $\beta$ blocker and calcium blocker combination therapy in patients with CPVT. Heart Rhythm 2010;7:S423.

45. Katz G, Khoury A, Kurtzwald E, et al. Optimizing catecholaminergic polymorphic ventricular tachycardia therapy in calsequestrin-mutant mice. Heart Rhythm 2010;7:1676-82.

46. Watanabe H, Chopra N, Laver D, et al.
Flecainide prevents catecholaminergic polymorphic ventricular tachycardia in mice and humans. Nat Med 2009;15:380-3.

47. van der Werf C, Kannankeril PJ, Sacher F, et al. Flecainide therapy reduces exerciseinduced ventricular arrhythmias in patients with catecholaminergic polymorphic ventricular tachycardia. J Am Coll Cardiol 2011;57:2244-54.

48. Zipes DP, Camm AJ, Borggrefe M, et al. ACC/AHA/ESC 2006 Guidelines for Management of Patients With Ventricular Arrhythmias and the Prevention of Sudden Cardiac Death: a report of the American College of Cardiology/American Heart Association Task Force and the European Society of Cardiology Committee for Practice Guidelines (writing committee to develop Guidelines for Management of Patients With Ventricular Arrhythmias and the Prevention of Sudden Cardiac Death): developed in collaboration with the European Heart Rhythm Association and the Heart Rhythm Society. Circulation 2006;114:e385-484.

49. Mohamed U, Gollob MH, Gow RM, Krahn AD. Sudden cardiac death despite an implantable cardioverter-defibrillator in a young female with catecholaminergic ventricular tachycardia. Heart Rhythm 2006;3: 1486-9.

50. Pizzale S, Gollob MH, Gow R, Birnie DH. Sudden death in a young man with catecholaminergic polymorphic ventricular tachycardia and paroxysmal atrial fibrillation. J Cardiovasc Electrophysiol 2008;19: 1319-21.

51. Sumitomo N, Sakurada H, Taniguchi K, et al. Association of atrial arrhythmia and sinus node dysfunction in patients with catecholaminergic polymorphic ventricular tachycardia. Circ J 2007;71:1606-9.

52. Sumitomo N, Nakamura T, Fukuhara J, et al. Clinical effectiveness of pulmonary vein isolation for arrhythmic events in a patient with catecholaminergic polymorphic ventricular tachycardia. Heart Vessels 2010;25:448-52.

53. Wilde AA, Bhuiyan ZA, Crotti L, et al. Left cardiac sympathetic denervation for catecholaminergic polymorphic ventricular tachycardia. N Engl J Med 2008;358:20249 .

54. Collura CA, Johnson JN, Moir C, Ackerman MJ. Left cardiac sympathetic denervation for the treatment of long QT syndrome and catecholaminergic polymorphic ventricular tachycardia using video-assisted thoracic surgery. Heart Rhythm 2009;6:752-9.

55. Makanjee B, Gollob MH, Klein GJ, Krahn AD. Ten-year follow-up of cardiac sympathectomy in a young woman with catecholaminergic polymorphic ventricular tachycardia and an implantable cardiovert- 
er defibrillator. J Cardiovasc Electrophysio 2009;20:1167-9.

56. Atallah J, Fynn-Thompson F, Cecchin F, et al. Video-assisted thoracoscopic cardiac denervation: a potential novel therapeutic option for children with intractable ventricular arrhythmias. Ann Thorac Surg 2008;86:1620-5.

57. Odero A, Bozzani A, De Ferrari GM, Schwartz PJ. Left cardiac sympathetic denervation for the prevention of life-threatening arrhythmias: the surgical supraclavicular approach to cervicothoracic sympathectomy. Heart Rhythm 2010;7:1161-5.

58. Hwang HS, Hasdemir C, Laver D, et al. Inhibition of cardiac $\mathrm{Ca} 2+$ release chan- nels (RyR2) determines efficacy of class I antiarrhythmic drugs in catecholaminergic polymorphic ventricular tachycardia. Circ Arrhythm Electro-physiol 2011;4:12835.

59. Kobayashi S, Yano M, Uchinoumi H, et al. Dantrolene, a therapeutic agent for malignant hyperthermia, inhibits catecholaminergic polymorphic ventricular tachycardia in a RyR2(R2474S/+) knock-in mouse model. Circ J 2010;74:2579-84.

60. Lehnart SE, Mongillo M, Bellinger A, et al. Leaky $\mathrm{Ca} 2+$ release channel/ryanodine receptor 2 causes seizures and sudden cardiac death in mice. J Clin Invest 2008;118: 2230-45.
61. Wehrens XH, Lehnart SE, Reiken SR, et al. Protection from cardiac arrhythmia through ryanodine receptor-stabilizing protein calstabin2. Science 2004;304:292-6.

62. Liu N, Ruan Y, Denegri M, et al. Calmodulin kinase II inhibition prevents arrhythmias in RyR2(R4496C+/-) mice with catecholaminergic polymorphic ventricular tachycardia. J Mol Cell Cardiol 2011;50: 214-22.

63. Tester DJ, Medeiros-Domingo A, Will ML, Ackerman MJ. Unexplained drownings and the cardiac channelopathies: a molecular autopsy series. Mayo Clin Proc 2011; 86:941-7. 